\title{
Omnidirectional Dual-Reflector Antenna with GO Shaped Main Reflector for Pattern Control in the Elevation Plane: OADC Case
}

\author{
José R. Bergmann ${ }^{1}$ and Fernando J. S. Moreira ${ }^{2}$ \\ ${ }^{1}$ CETUC_Center for Telecommunications Studies, Catholic University of Rio de Janeiro, 22453-900 Rio de Janeiro, RJ, Brazil \\ ${ }^{2}$ Department of Electronics Engineering, Federal University of Minas Gerais, 31270-901 Belo Horizonte, MG, Brazil
}

Correspondence should be addressed to José R. Bergmann, bergmann@cetuc.puc-rio.br

Received 4 December 2011; Revised 29 February 2012; Accepted 29 February 2012

Academic Editor: Shyh-Kang Jeng

Copyright ( 2012 J. R. Bergmann and F. J. S. Moreira. This is an open access article distributed under the Creative Commons Attribution License, which permits unrestricted use, distribution, and reproduction in any medium, provided the original work is properly cited.

\begin{abstract}
This paper presents a formulation for shaping the main reflector of an axis-symmetric dual-reflector antenna designed to offer an omnidirectional coverage with an arbitrary radiation pattern in the vertical plane. The subreflector is generated by an axisdisplaced conic, and the main reflector is shaped to achieve a prescribed far-field radiation pattern. The procedure is based on geometrical optics (GO) principles. Two distinct far-field ray structures are explored and their limitations are identified. The GO shaping results are validated by analysis provided by the accurate method of moments technique.
\end{abstract}

\section{Introduction}

Local multipoint distribution systems (LMDSs) represent a radio-based access technology with cellular architecture offering flexible telecommunication systems with possibility of application for digital interactive TV that will stimulate growth of e-commerce, telemedicine, and so forth. For operation at millimeter waves, axis-symmetric omnidirectional dual-reflector antennas may lead to compact designs and yield the wide frequency bandwidth required to operate as an LMDS base station. The reflectors can be shaped to provide a cosecant-squared radiation pattern in the vertical plane and radiate an almost uniform power density over the coverage area. For vertical polarization, several designs have been investigated [1-5].

Based on GO, a simple shaping procedure for dualreflector omnidirectional antennas is presented in [5]. The generating curve of the axis-symmetric subreflector is an ellipse (omnidirectional axis-displaced ellipse-OADE type) and the shaped axis-symmetric main reflector defines the antenna radiation pattern in the elevation plane. Here, we explore dual-reflector antennas of the OADC (omnidirectional axis-displaced Cassegrain) type, where the subreflector has a virtual annular caustic and the main-reflector generatrix is shaped to control the far-field radiation in the vertical (elevation) plane. In Section 2, geometrical features of the OADC configuration are briefly discussed. In Section 3 the design of the OADC subreflector surface is discussed. In Section 4 the GO main-reflector shaping is described. To identify potentialities and limitations of the OADC configuration, Section 5 presents design examples where the main reflector is shaped to provide a cosecant-squared radiation pattern in the elevation plane. All case studies are validated by method-of-moments (MoMs) simulations, which account for all electromagnetic effects present on the reflector and horn structure [5].

\section{Basic Geometrical Features}

The OADC antenna is composed of two axis-symmetric reflectors with a common symmetry axis (the $z$-axis), as in Figures 1(a) and 1(b). Its main features are discussed in [35]. The subreflector is generated by a conic section with two focci located at points $O$ (the feed phase center) and $P$, so that a ray emanating from $O$ is reflected towards $P$, which defines the virtual annular caustic of the subreflector. As illustrated 


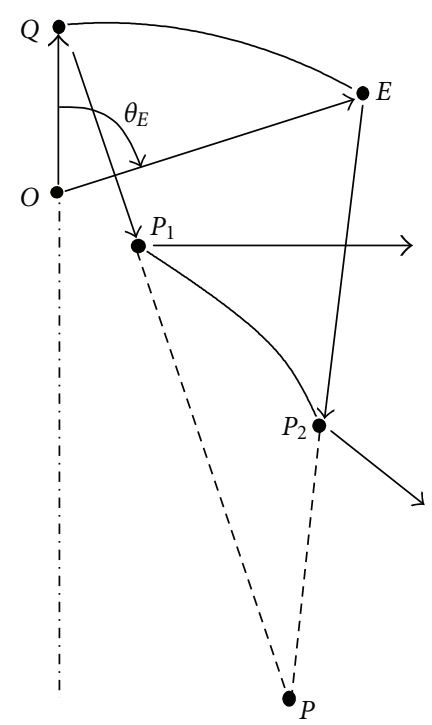

(a)

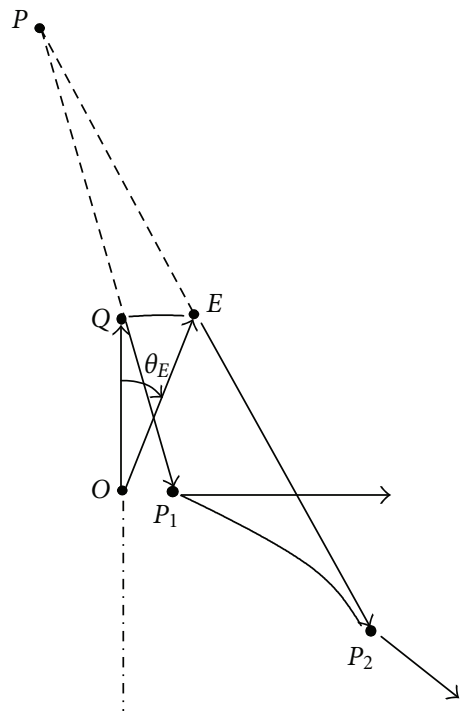

(b)

FIGURE 1: Some different configurations for the generating curves of OADC sub- and main-reflectors.

in Figures 1(a) and 1(b) the conic section that generates the subreflector depends on the location of $P$ : it is an ellipse when $P$ appears below the main reflector, a hyperbola when $P$ is above the subreflector, or a parabola when $P$ tends to infinity. The main reflector is generated by a shaped curve limited by points $P_{2}$ and $P_{1}$, which define the main-reflector inner and outer edges, respectively. $P_{2}$ is located out of the symmetry axis, leaving space for the feed access to focus $O$ (Figure 2). The feed has a circularly symmetric radiation pattern $G_{F}\left(\theta_{F}\right)$, where $\theta_{F}$ is the angle between the feed ray and the $z$-axis. From GO principles, the main reflector generatrix is shaped so that rays coming from $P$ are reflected toward the far-field region to attend a specified circularly symmetric radiation pattern $G_{A}(\theta)$, where $\theta$ is the angle between the ray reflected by the main reflector and the $z$-axis. The GO transformation $G_{F}\left(\theta_{F}\right) \rightarrow G_{A}(\theta)$ can be obtained by two main-reflector ray structures, one with a real and another with a virtual caustic in the elevation plane, which lead to different main-reflector designs, as discussed in Section 5.

\section{OADC Subreflector Design}

The conic that generates the subreflector must be established first, in order to define the mapping $\theta_{F} \rightarrow \theta_{S}$, where $\theta_{S}$ is the angle between the ray reflected by the subreflector and the $z$-axis (Figure 2). That can be accomplished by closely following the formulation of the OADE subreflector design discussed in Section 3 of [5]. However, as the subreflector ring caustic is virtual in the OADC configuration, the reverse of the feed radiation does not occur and the feed principal ray $\left(\theta_{F}=0\right)$ is reflected toward $P_{2}$ with $\theta_{S}=\theta_{S 0}$ (Figure 2 ). Consequently, (8) of [5] is substituted by

$$
\eta_{S 0}=\cot \left(\frac{\theta_{S 0}}{2}\right)=\frac{-\varepsilon \sin \beta}{\varepsilon \cos \beta-1},
$$

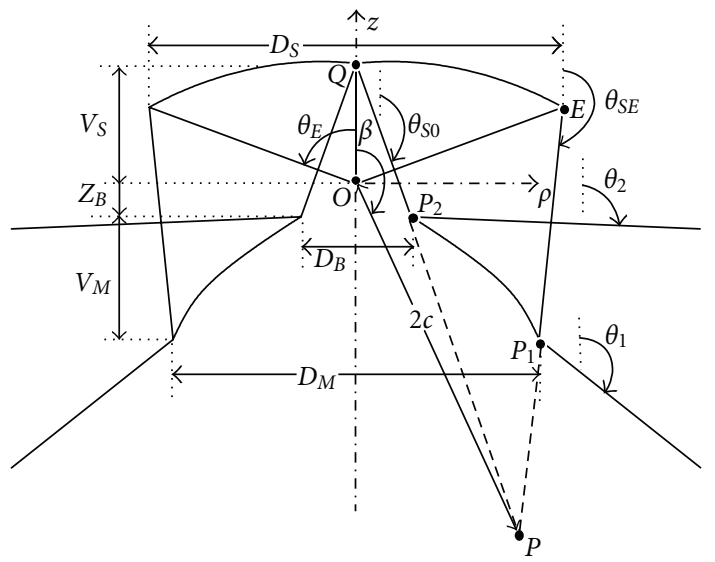

FIGURE 2: Basic geometry of the OADC antenna with shaped main reflector.

where $\varepsilon$ is the eccentricity and $\beta$ is the axis angular tilt (Figure 2) of the subreflector conic. That leads to:

$$
\begin{gathered}
\varepsilon \sin \beta=\frac{2 D_{S} \eta_{S 0}}{D_{S}\left(1-\eta_{E}^{2}+2 \eta_{E} \eta_{S 0}\right)+4 V_{S} \eta_{E}}, \\
\varepsilon \cos \beta=1-\left[\frac{2 D_{S}}{D_{S}\left(1-\eta_{E}^{2}+2 \eta_{E} \eta_{S 0}\right)+4 V_{S} \eta_{E}}\right],
\end{gathered}
$$

which substitute (9) and (10) of [5], respectively. In (2) and (3), $D_{S}$ is the subreflector diameter, $V_{S}$ is the distance between $O$ and the subreflector vertex, $\eta_{E}=\cot \left(\theta_{E} / 2\right)$, and $\theta_{E}$ is the subreflector edge angle (see Figure 2). The value of $\eta_{S 0}$ necessary to evaluate (2) and (3) is (see Figure 2):

$$
\eta_{S 0}=-\left(\frac{V_{S}-z_{B}}{D_{B} / 2}\right)+\sqrt{\left(\frac{V_{S}-z_{B}}{D_{B} / 2}\right)^{2}+1},
$$


where $D_{B}$ and $z_{B}$ locate $P_{2}$. After determining the conic parameters, the subreflector surface is described by (2) of [5] and the mapping $\theta_{F} \rightarrow \theta_{S}$ is given by (4) of [5].

\section{OADC Main-Reflector Shaping}

The GO shaping of the OADC main reflector is similar to that of the OADE in [5]. The main reflector is represented by a real function $L\left(\eta_{S}\right)$ and a point at this surface is located by $[6]$ :

$$
\vec{r}_{M}\left(\theta_{S}\right)=\left\lfloor\rho_{P} \mp 2 \eta_{S} e^{L\left(\eta_{S}\right)}\right\rfloor \hat{\rho}+\left\lfloor z_{P} \mp\left(\eta_{S}^{2}-1\right) e^{L\left(\eta_{S}\right)}\right\rfloor \hat{z},
$$

where $\rho_{P}$ and $z_{P}$ are the coordinates of the subreflector annular caustic $P$ [5], $\eta_{S}=\cot \left(\theta_{S} / 2\right)$, and the negative or positive sign depends whether $P$ is below the main reflector (with $\beta>0$, as in Figure 1(a)) or above the subreflector $(\beta<0$, as in Figure 1(b)), respectively. By applying the law of reflection at the main reflector, the relation between the directions $\theta_{S}$ and $\theta$ is given by [5]:

$$
\frac{\partial L\left(\eta_{S}\right)}{\partial \eta_{S}}=\frac{2}{\eta-\eta_{S}}
$$

where $\eta=\cot (\theta / 2) . L\left(\eta_{S}\right)$ is determined from the integration of (6), numerically evaluated in the domain $0 \leq \theta_{F} \leq \theta_{E}$ with the help of the relation between $\theta_{F}$ and $\theta_{S}$ (equation (4) of [5]). However, one still needs the relation between $\theta_{F}$ and $\theta$, which is obtained by imposing the conservation of energy flow within a pencil of rays departing from $O$ and leaving the main reflector after both reflections. The rays emerging from $O$, confined inside the cone $\theta_{F} \leq \theta_{E}$, are finally reflected by the main reflector between $\theta_{2}$ and $\theta_{1}$ (see Figure 1 ). If $\theta_{2}<\theta_{1}$ the main reflector has a virtual caustic in the elevation plane. Otherwise, the caustic is real (e.g., Figure 6). The conservation of energy establishes that:

$$
\int_{0}^{\theta_{F}} G_{F}\left(\theta_{F}^{\prime}\right) \sin \theta_{F}^{\prime} d \theta_{F}^{\prime}=N \int_{\theta_{2}}^{\theta} G_{A}\left(\theta^{\prime}\right) \sin \theta^{\prime} d \theta^{\prime},
$$

where $N$ is a normalization factor obtained by considering $\theta_{F}=\theta_{E}$ and $\theta=\theta_{1}$. Equation (7) provides the relation between $\theta_{F}$ and $\theta$ as a function of the prescribed radiation patterns $G_{F}\left(\theta_{F}\right)$ and $G_{A}(\theta)$.

\section{OADC Case Studies}

We now present OADC designs suited to operate as basestation antennas in point-multipoint radio systems. In all cases investigated, (6) was numerically evaluated by a fourthorder Runge-Kutta method. As in [5, 7], the main reflectors were shaped to radiate a cosecant-squared pattern in the elevation plane, given by:

$$
G_{A}(\theta)=G_{A O} \csc ^{2}\left(\theta-\frac{\pi}{2}\right), \quad \text { for } \theta \in\left[\theta_{2}, \theta_{1}\right]
$$

where $G_{A O}$ is a normalization factor. $G_{A}(\theta)$ of (8) provides a uniform coverage on ground and the limits $\theta_{2}$ and $\theta_{1}$ (see Figure 2) are defined to avoid interference in adjacent
TABLE 1: Design parameters of Cases I-IV.

\begin{tabular}{lcccc}
\hline Case & I & II & III & IV \\
\hline$V_{S}(\lambda)$ & 13.6 & 13.6 & 13.6 & 13.6 \\
$D_{S}(\lambda)$ & 36.05 & 36.05 & 36.05 & 36.05 \\
$D_{M}(\lambda)$ & 38.18 & 38.38 & 38.1 & 38.2 \\
$V_{M}(\lambda)$ & 22.78 & 25.62 & 21.45 & 23.02 \\
$\varepsilon$ & 0.9214 & 0.9214 & 0.9214 & 0.9214 \\
$\beta$ & $174.7^{\circ}$ & $174.7^{\circ}$ & $174.7^{\circ}$ & $174.7^{\circ}$ \\
$2 c(\lambda)$ & 318.31 & 318.31 & 318.31 & 318.31 \\
$\theta_{2}$ & $92^{\circ}$ & $92^{\circ}$ & $135^{\circ}$ & $150^{\circ}$ \\
$\theta_{1}$ & $135^{\circ}$ & $150^{\circ}$ & $92^{\circ}$ & $92^{\circ}$ \\
$V_{O}$ & 39.1 & 42.3 & 37.5 & 40.5 \\
$G_{\mathrm{MAX}}$ & 11.04 & 11.12 & 11.8 & 12.06 \\
\hline
\end{tabular}

communication systems. Aiming vertical polarization, the OADC antennas were fed by the same TEM coaxial horn model adopted in [5]. In all MoM simulations, the horn structure (Figure 3 of [5]) was analyzed together with both reflectors to account for mutual-coupling effects in the antenna radiation pattern. However, in the GO synthesis $G_{F}\left(\theta_{F}\right)$ of (7) was modeled by (22) of [5]. The horn diameter is relatively small (about $2.4 \lambda$ ). So, the feed phase center is close to the horn's aperture, placed at the OADC focal plane. To obtain compact dual-reflector arrangements, the mainreflector central edge $P_{2}$ is always at the focal plane (i.e., $z_{B}=0$ with $D_{B}=2.4 \lambda$ ).

In the following examples, the antenna dimensions adopted in [7] were used as references to explore antenna designs for different coverage angles $\left(\theta_{2}, \theta_{1}\right)$ and identify limitations imposed by the ray structure emerging from the main reflector. Reference [7] presents an omnidirectional dual-reflector antenna where the subreflector is a paraboloid and the main reflector is GO shaped to provide a cosecantsquared radiation pattern in the vertical plane from $\theta_{2}=92^{\circ}$ to $\theta_{1}=150^{\circ}$ (as $\theta_{2}<\theta_{1}$ the main reflector has a virtual caustic in the elevation plane). The sub- and main-reflector diameters are $D_{M}=D_{S}=38 \lambda$, the subreflector focal distance $V_{S} \approx 13.6 \lambda$ and the main-reflector height $V_{M} \approx 26.4 \lambda$. The antenna radiation pattern has a directivity peak of $11.2 \mathrm{dBi}$ and oscillates $\pm 3 \mathrm{~dB}$ around the desired cosecant-squared pattern [7].

5.1. Designs with Virtual-Caustic Main Reflector. In the first example (Case I), the main reflector was shaped to radiate a cosecant-squared pattern similar to that of [7], but from $\theta_{2}=92^{\circ}$ to $\theta_{1}=135^{\circ}$. The subreflector edge angle was set $\theta_{E}=65^{\circ}$ to minimize spillover above the horizon. For comparative purposes, $D_{S}=36.05 \lambda$ was chosen in order to generate a shaped OADC antenna with $V_{M}, D_{M}$, and $V_{S}$ (their values are listed in Table 1) similar to the antenna dimensions in [7]. With the help of the formulation presented in Section 3, the subreflector generating ellipse was determined $\left(\varepsilon=0.9214,2 c=318.31 \lambda\right.$, and $\left.\beta=174.7^{\circ}\right)$, with the subreflector caustic $P$ located below the main reflector $\left(\rho_{P}=29.4 \lambda\right.$ and $\left.z_{P}=-316.9 \lambda\right)$. Figure 3 illustrates 


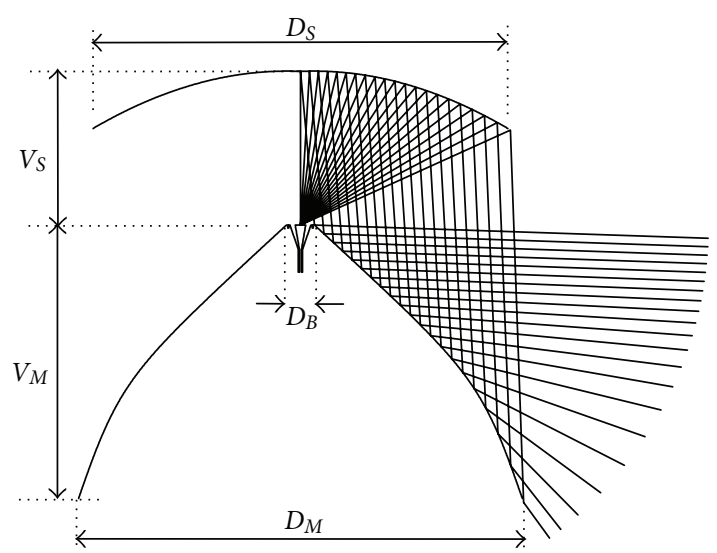

FIgURE 3: Sub- and main-reflector generatrices of the OADC antenna of Case I.

the shaped antenna geometry and the ray structure throughout the dual-reflector system. The subreflector generating ellipse has a large inter-focal distance $2 c$ that leads to reflected rays almost parallel to each other between the reflectors. Table 1 lists values of some relevant parameters of Case I, together with an approximate estimate of the antenna volume $\left(V_{O}\right)$.

As the GO synthesis does not take into account diffraction and coupling effects among feed and reflectors, a fullwave analysis based on the method of moments (MoMs) was conducted to validate the shaping procedure, including the coaxial horn structure. Figure 4 shows the radiation pattern in the vertical plane (solid line), together with the desired cosecant-squared pattern (dashed line) and its $\pm 3 \mathrm{~dB}$ limits (dotted lines). The radiation pattern is confined within the $\pm 3 \mathrm{db}$ limits, with a peak $G_{\mathrm{MAX}}=11.04 \mathrm{dBi}$ at $\theta \approx 94^{\circ}$.

To explore the design for a wider angular region, in Case II the main reflector was shaped to radiate a cosecantsquared pattern from $\theta_{2}=92^{\circ}$ to $\theta_{1}=150^{\circ}$, as in [7]. The subreflector is the same employed in Case I. When compared with Case I, the increase of the coverage angle $\theta_{1}$ does not significantly affect the overall antenna dimensions, as one may inspect from Table 1 . The antenna geometry is basically that illustrated in Figure 3 for Case I, except that now the mainreflector edge $P_{1}$ is pushed downwards by about $3 \lambda\left(V_{M}=\right.$ $25.62 \lambda$ ), as a consequence of the near grazing incidence upon that region. Consequently, it is expected a higher inaccuracy of the GO shaping at that region. As can be observed in Figure 5, the MoM radiation pattern of Case II has a slightly higher radiation peak $\left(G_{\mathrm{MAX}}=11.12 \mathrm{dBi}\right)$ and a performance similar to the design of [7]. When compared with Case I, the coverage extension up to $\theta_{1}=150^{\circ}$ yields higher oscillations around the specified cosecant-squared pattern, with levels outside the $\pm 3 \mathrm{~dB}$ limits.

5.2. Designs with Real-Caustic Main Reflector. In an alternative design (Case III), the same subreflector of Cases I and II was employed together with a main reflector that was shaped to radiate a cosecant-squared pattern $G_{A}(\theta)$, but from $\theta_{2}=$ $135^{\circ}$ to $\theta_{1}=92^{\circ}$ (now the main reflector has a real caustic

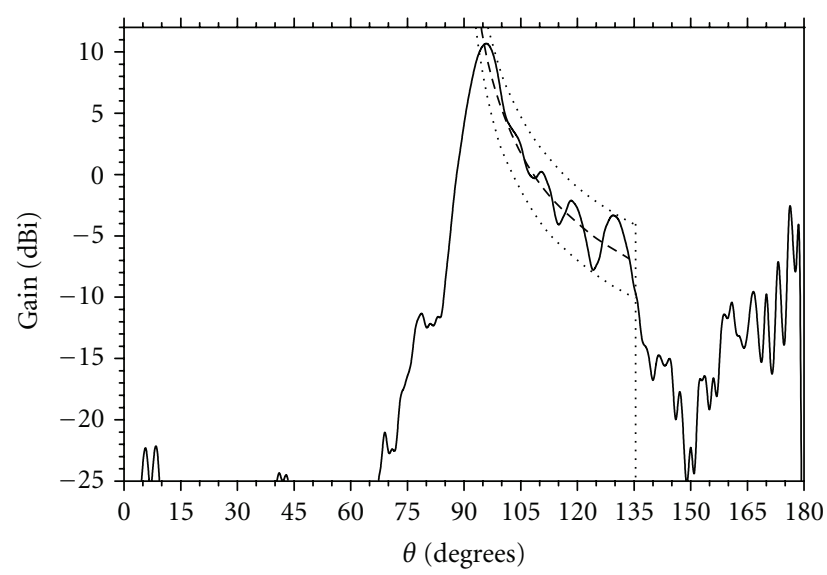

FIgURE 4: MoM radiation pattern in the elevation plane of the OADC antenna of Case I (solid line), together with the cosecantsquared pattern (dashed line) and its $\pm 3 \mathrm{~dB}$ limits (dotted lines).

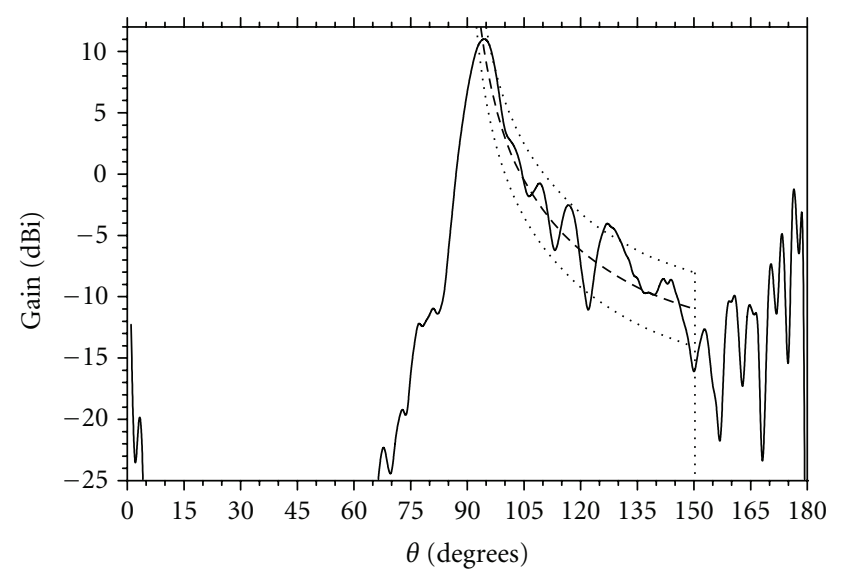

FIGURE 5: MoM radiation pattern in the elevation plane of the OADC antenna of Case II (solid line), together with the cosecantsquared pattern (dashed line) and its $\pm 3 \mathrm{~dB}$ limits (dotted lines).

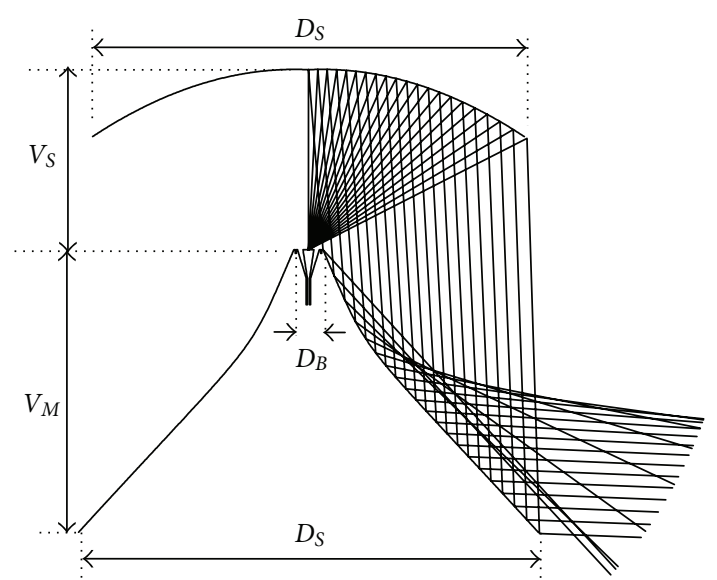

FIgURE 6: Sub- and main-reflector generatrices of the Case III OADC antenna. 


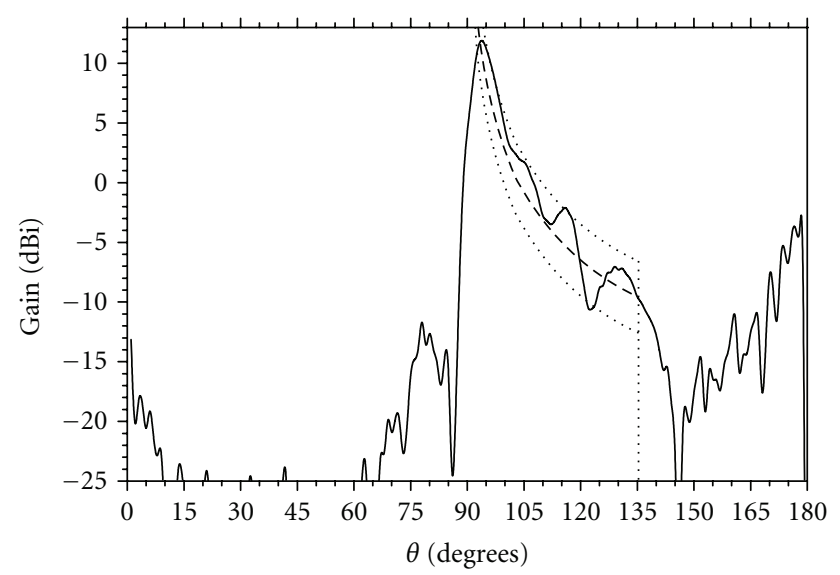

Figure 7: MoM radiation pattern in the elevation plane of the OADC antenna of Case III (solid line), together with the cosecantsquared pattern (dashed line) and its $\pm 3 \mathrm{~dB}$ limits (dotted lines).

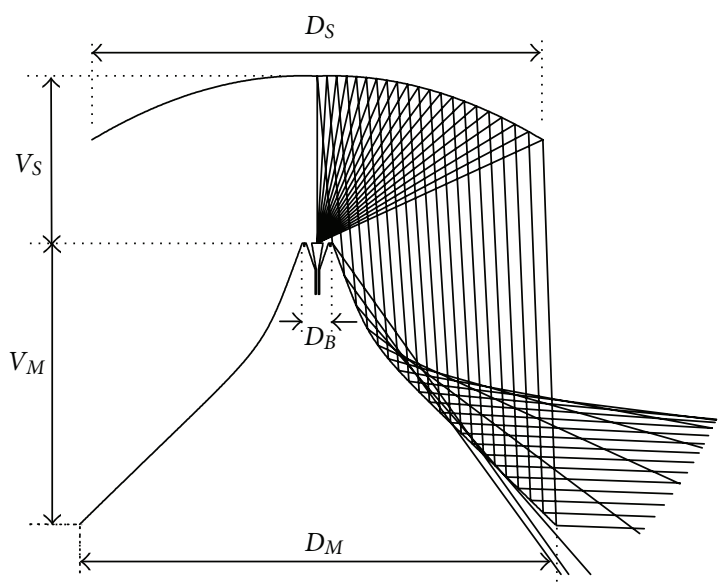

FIgURE 8: Sub- and main-reflector generatrices of the Case IV OADC antenna.

in the elevation plane). The values of relevant antenna dimensions are listed in Table 1. Figure 6 depicts the dualreflector configuration obtained from the GO synthesis, where the main-reflector real caustic is apparent. From Figure 6 one observes that the real caustic is closer to the upper portion of the main reflector and that there is a significant clearance (about $3 \lambda$ ) between the main-reflector rim $P_{1}$ and the ray reflected from the upper portion with $\theta=\theta_{2}$. Figure 7 shows the MoM radiation pattern obtained for Case III, which has a peak $G_{\mathrm{MAX}}=11.8 \mathrm{dBi}$ at $\theta \approx 93^{\circ}$ and is almost completely confined within the $\pm 3 \mathrm{db}$ limits of the cosecant-squared pattern.

Although Case III is free of blockage (see Figure 6), the real caustic in front of the main reflector may impose limitations for designs with larger angular coverages in the elevation plane. To illustrate this, in Case IV we have repeated the shaping conducted in Case III, but with $\theta_{2}$ increased to $150^{\circ}$ (i.e., the angular coverage in the elevation plane is similar to that of Case II). The shaped antenna configuration is illustrated in Figure 8, from which one inspects that the main-reflector rim blocks some rays reflected from the upper portion of the main reflector, leaving part of the coverage area in the shadow, in the optical sense. That imposes a limitation on the far-field coverage, where the angular limit $\theta_{2}<\theta_{L}$ to avoid the main-reflector blockage is given by (see Figure 2):

$$
\theta_{L}=\pi-\arctan \left(\frac{D_{M}-D_{B}}{2\left(V_{M}+z_{B}\right)}\right),
$$

where $\theta_{L}$ is the $\theta$ angle of the straight segment from $P_{2}$ to $P_{1}$. From the values listed in Table $1, \theta_{L}=142.1^{\circ}$ for Case IV.

With the help of Figures 3 and 6 and Table 1, one observes that the configuration with a real main-reflector caustic (Case III) has about the same dimensions and volume $\left(V_{O}\right)$ of the antenna with a virtual main-reflector caustic (Case I). On the opposite side, when compared with the OADE designs investigated in [5] for similar specifications, the choice of the type of ray structure in front of the main reflector can affect drastically the overall dimensions of the reflector antenna. As observed in Figure 4 of [5], the virtual caustic option leads to a larger shaped main reflector as the ray coming from the subreflector vertex, point $Q$ (see Figure 2), impinges upon the main reflector at an almost grazing incidence, pushing the reflector edge downwards. Consequently, it yields a virtual caustic design with an antenna volume larger than twice of the real caustic one.

\section{Conclusions}

A GO procedure for the main-reflector shaping of an omnidirectional ADC antenna was presented. The body-ofrevolution OADC configuration is composed of a subreflector generated by an axis-displaced conic section and a mainreflector generated by a shaped curve to control the radiation pattern in the elevation plane. The procedure was employed in the shaping of two different OADC arrangements: with virtual or real main-reflector caustic in the elevation plane. Four designs were presented and analyzed by an accurate MoM technique. For each case, the far-field and geometrical limitations were outlined, such as the conditions to avoid the main-reflector self-blockage that would leave part of the coverage area in the shadow, in the optical sense. It was also verified that OADC antennas with a virtual main-reflector caustic are appropriate choices for designs with compact dimensions.

\section{Acknowledgment}

This work was partially supported by CNPq, Brazil, under Projects 573939/2008-0, 304953/2009-1, 304953/2009-1, 470064/2011-0, and 554661/2010-1 and FAPEMIG, Brazil.

\section{References}

[1] A. Norris and W. Waddoup, "A millimetric wave omnidirectional antenna with prescribed elevation shaping," in Proceedings of the 4th International Conference on Antennas and Propagation (ICAP'85), pp. 141-145, 1985. 
[2] A. Pino, A. Acuña, and J. Lopez, "An omnidirectional dualshaped reflector antenna," Microwave and Optical Technology Letters, vol. 27, no. 5, pp. 371-374, 2000.

[3] J. Bergmann and F. Moreira, "An omni directional ADE reflector antenna," Microwave and Optical Technology Letters, vol. 40 , no. 3, pp. 250-254, 2004.

[4] F. J. da Silva Moreira and J. R. Bergmann, "Axis-displaced dualreflector antennas for omnidirectional coverage with arbitrary main-beam direction in the elevation plane," IEEE Transactions on Antennas and Propagation, vol. 54, no. 10, pp. 2854-2861, 2006.

[5] J. R. Bergmann and F. J. S. Moreira, "Omnidirectional ADE antenna with a GO-shaped main reflector for an arbitrary farfield pattern in the elevation plane," IET Microwaves, Antennas and Propagation, vol. 3, no. 7, pp. 1028-1035, 2009.

[6] B. S. Westcott, F. A. Stevens, and F. Brickell, "GO synthesis of offset dual reflectors," IEE Proceedings $H$, vol. 128, no. 1, pp. 11-18, 1981 .

[7] P. Besso, R. Bills, P. Brachat, and R. Vallauri, "Millimetric wave omnidirectional antenna with cosecant squared elevation pattern," in Proceedings of the 10th International Conference on Antennas and Propagation (ICAP '97), pp. 448-451, April 1997. 

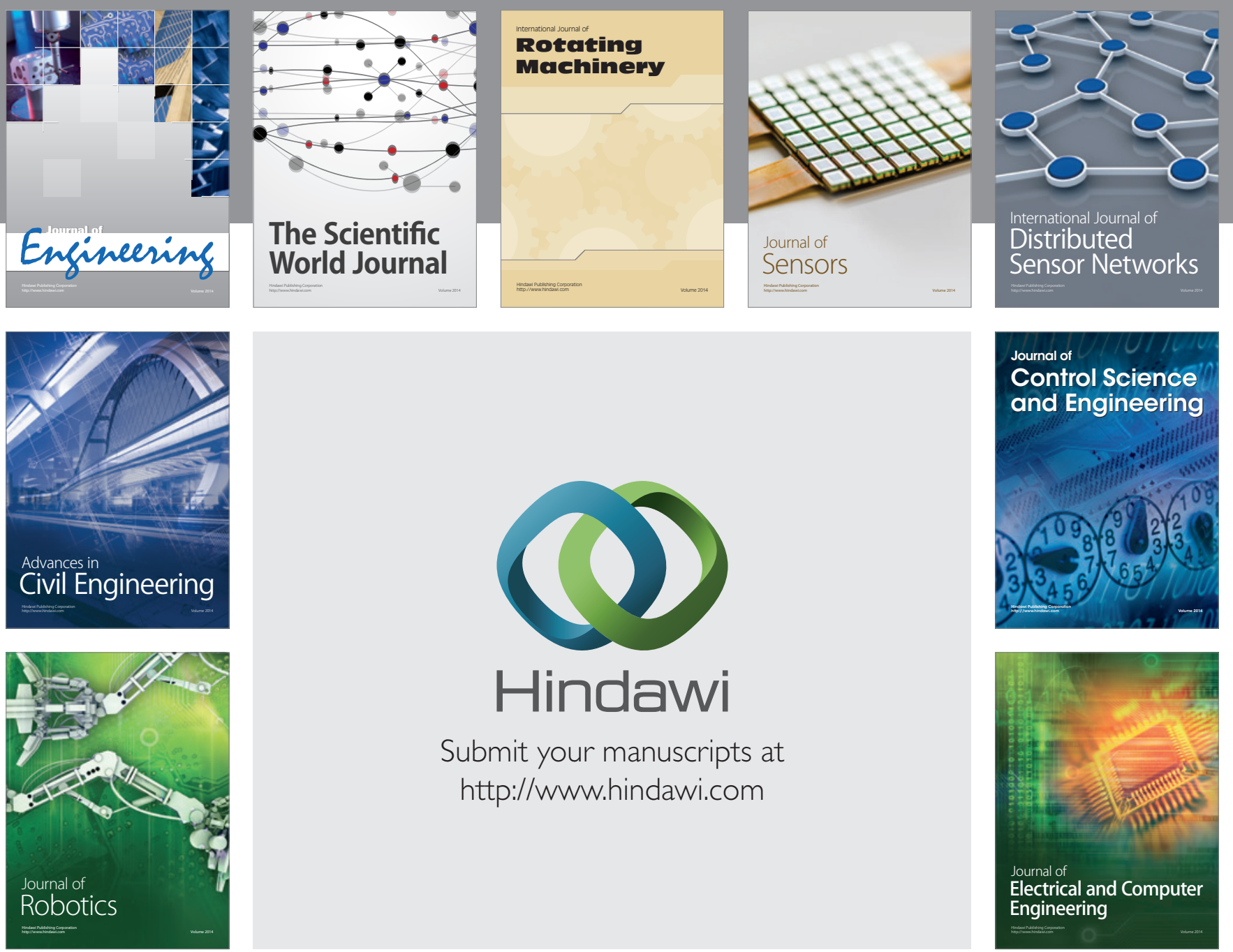

Submit your manuscripts at

http://www.hindawi.com
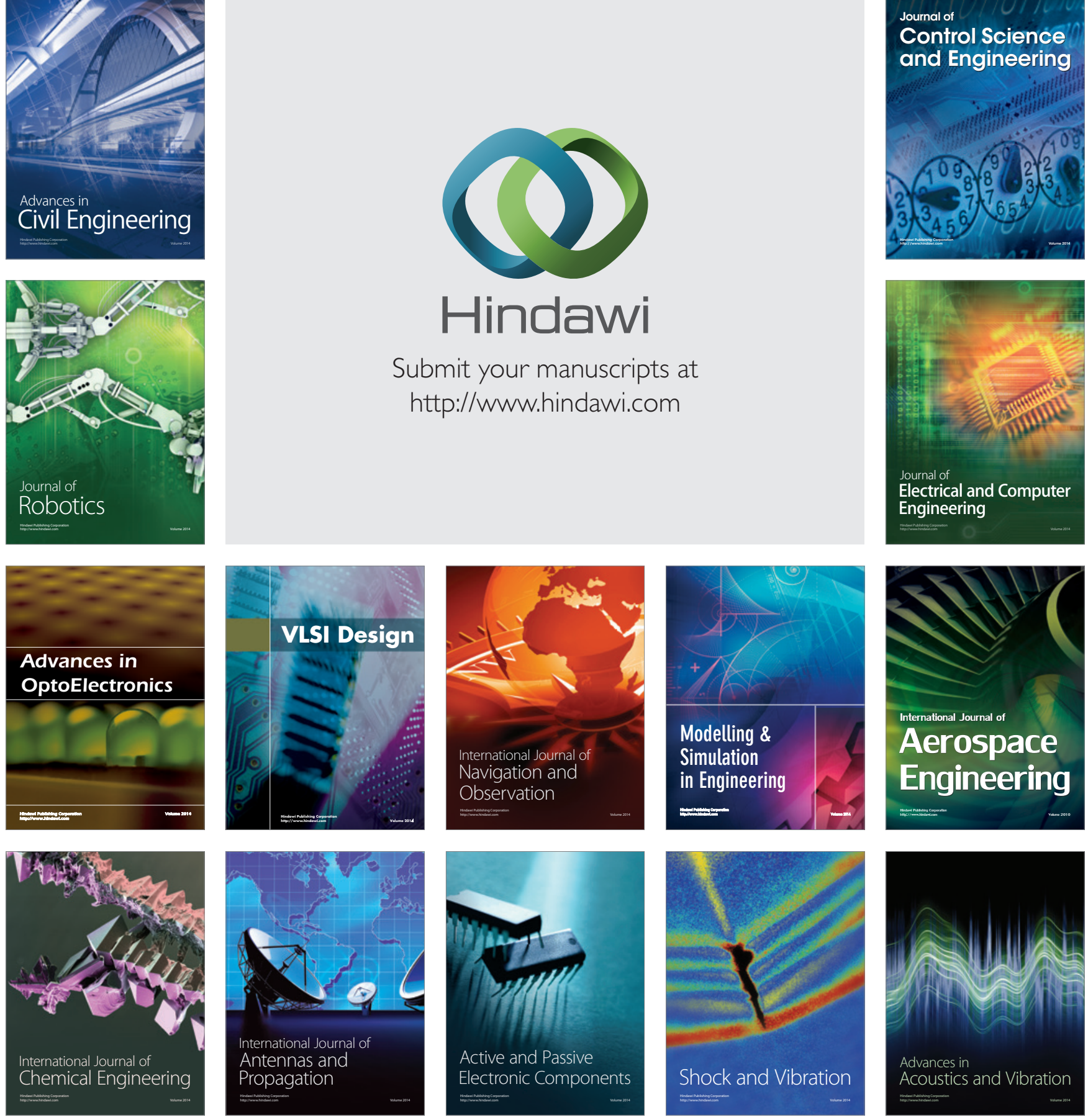